\title{
Near-equilibrium dynamics of Doi models for liquid crystal polymer flows: catastrophic and regularized behavior
}

\author{
M. Gregory Forest* \\ Department of Mathematics \\ The University of North Carolina at Chapel Hill \\ Chapel Hill, NC 27599-3250 \\ and \\ Qi Wang ${ }^{\dagger}$ \\ Department of Mathematical Sciences \\ Indiana University-Purdue University Indianapolis \\ Indianapolis, IN 46202
}

\begin{abstract}
Doi models for flows of concentrated solutions of homogeneous liquid crystal polymers (LCPs) are analyzed in the quadratic closure approximation. Our purpose is to clarify a remarkable near-equilibrium behavior of these equations which has apparently gone unnoticed; these results are important for any numerical or experimental interpretations of LCP flows based on Doi models near mechanical and nematic equilibria. To reveal this behavior, we analytically solve the linearized Doi nematodynamic equations; this calculation explicitly captures the coupling between the pure nematic and pure hydrodynamic linearized dynamics. The original Doi model without solvent viscosity is analyzed first: the low concentration $(N<3)$ isotropic phase and the high concentration $\left(N>\frac{8}{3}\right)$ prolate nematic phase yield well-posed linearized dynamics; at higher concentration both the isotropic phase $(N>3)$ and the oblate nematic phase $(N>3)$ yield catastrophic linearized dynamics, with exponential growthrates proportional to the amplitude of the wavevector of the linearized disturbance. This result implies there is unbounded growth in vanishingly small lengthscales for data near these equilibria. We then explore three physical regularizations of the original Doi model: solvent viscosity, finite-range intermolecular interactions, and spatial inhomogeneity in LCP concentration. Each effect yields well-posed linearized dynamics of
\end{abstract}

*Email: forest@math.unc.edu.

${ }^{\dagger}$ Email: qwang@math.iupui.edu 
all flow-nematic equilibria, with bounded growth rates. Solvent viscosity and spatial inhomogeneity alone are not sufficient to produce a finite wavelength instability cutoff of the high-concentration isotropic and oblate nematic equilibria, whereas a finite-range intermolecular potential alone yields a finite cutoff. The flow-orientation interactions for unstable nematic phases produce a spatial wavevector dependence of the instability, from which we reveal a flow-induced spatially anisotropic (or directional) instability of the oblate phase.

Keywords: Liquid crystals, flow stability, ill-posedness, non-Newtonian flows.

\section{Introduction}

The original Doi model [4] for flows of homogeneous liquid crystal polymers, and recent generalizations, have been heavily studied and applied for more than a decade. The Doi theory has been used to model many interesting LCP flow phenomena such as tumbling, log-rolling, wagging $[12,14,17,3,18,16]$ and reduced capillary instability of LCP filaments [7]. Recently, a Doi model has been used in complex flow simulations to reproduce fascinating orientation patterns [6], and to predict surface and centerline velocity reversal in fiber spinning processes [15]. In other applications, the stability of nematic equilibrium phases in the presence of an imposed flow field has been actively pursued $[3,17,2,18,16,10,7]$.

Recently, the authors discovered that the original Doi model predicts unusual, indeed pathological, behavior with regard to Rayleigh capillary instability when the nematic LCP obeys two conditions: the equilibrium microstructure is either in the isotropic or the uniaxial oblate nematic phase; and the polymer concentration is sufficiently high. The unusual result is that the linearized hydrodynamic-nematodynamic free surface equations admit an infinitedimensional unstable manifold for such equilibrium axisymmetric LCP filaments.

The quick description of this unphysical behavior is that the original Doi model can be viewed as a coupling of anisotropic microstructural dynamics to the inviscid fluid equations. The classical inviscid analysis of Rayleigh shows there is a capillary instability that is onedimensional, with linearized growthrate that can be exactly calculated in closed form; all other linearized modes are neutrally stable. When viscosity is added to the fluid equations, the one-dimensional capillary instability survives, but the neutrally stable dimensions are all stabilized by viscosity. Our analysis of the original Doi equations without solvent viscosity [7] is quite similar to the viscous fluid analysis, except that the nematic coupling (in contrast to viscosity) destabilizes the neutrally stable modes for certain nematic phases (the high density isotropic and oblate nematic phases); yet stabilizes the low density isotropic and high density prolate nematic phases. The result is that the Doi nematodynamic equations without solvent viscosity imply some free surface flow-nematic equilibria are infinite-dimensionally unstable. This troubling result requires further understanding of the Doi model equations, apart from the complications of the free surface and associated capillary instability. That is the motivation for the present paper, in which we explain the source of this pathological stability prediction in the original Doi model. We then show that recent physical generalizations of the Doi model, incorporating solvent viscosity or nonlocal diffusive effects, arrest this unphysical behavior. 
Nonetheless, these results serve as a cautionary tale with regard to any predictions made from LCP flow models nearby the original Doi model. We note that the catastrophic behavior in the analysis to follow and in the capillary instability analysis occurs if and only if the orientation equilibrium phase is unstable within the pure orientation dynamics, independent of flow. The unstable orientation behavior then couples to the fluid equations through the polymer (orientation) stress contribution, and the Doi nematodynamic equations without solvent viscosity or without long-range intermolecular effects cannot control the coupling of the orientational instability to the inviscid fluid dynamics.

\section{Mathematical Formulation}

We first review the approximate Doi theory for spatially homogeneous LCPs obtained from the quadratic closure approximation following Bhave et al. [2]. The intermolecular interaction in the theory is characterized by the Maier-Saupe short-range excluded-volume potential:

$$
V_{M-S}(\mathbf{m})=-\frac{3 N B T}{2} \mathbf{Q}: \mathbf{m m}
$$

Here $\mathbf{Q}$ is a symmetric, traceless, second-order orientation tensor defined through the second moment of the unit vector $\mathbf{m}$ in the rodlike (dumbbell) molecular direction, averaged with respect to a normalized probability density function $f(\mathbf{m}, \mathbf{x}, t)$ at space location $\mathbf{x}$ and time $t$ :

$$
\begin{aligned}
& \mathbf{Q}=\langle\mathbf{m} \mathbf{m}\rangle-\frac{\mathbf{I}}{3}, \\
& \langle(\bullet)\rangle=\int_{\|\mathbf{m}\|=1}(\bullet) f(\mathbf{m}, \mathbf{x}, t) d \mathbf{m},
\end{aligned}
$$

$N$ is a dimensionless measure of the polymer concentration which also characterizes the strength of the intermolecular potential, $B$ is the Boltzmann constant and $\mathrm{T}$ is absolute temperature. The governing equations in the approximate theory for flows of LCPs consist of the constitutive equation which couples flow and orientation contributions to the stress, the evolutionary equation for the orientation tensor, together with the conservation of linear momentum and incompressibility condition.

Conservation of linear momentum:

$$
\rho \frac{d}{d t} \mathbf{v}=\nabla \cdot \tau+\mathbf{b}
$$

where $\rho$ is the density of the LCP liquid, $\mathbf{v}$ is the velocity field, $\tau$ is the total stress tensor, $\mathbf{b}$ is the external body force, which will be neglected in this paper since our focus is on simple behavior near flow equilibrium, and $\frac{d}{d t}(\bullet)$ denotes the material derivative: $\frac{\partial}{\partial t}+\mathbf{v} \cdot \nabla$.

Incompressibility:

$$
\nabla \cdot \mathbf{v}=0
$$


Constitutive equation for the stress:

$$
\left\{\begin{array}{l}
\tau=-p \mathbf{I}+\hat{\tau}, \\
\hat{\tau}=2 \eta \mathbf{D}+3 n B T\left[\left(1-\frac{1}{3} N\right) \mathbf{Q}-N(\mathbf{Q} \cdot \mathbf{Q})+N(\mathbf{Q}: \mathbf{Q})\left(\mathbf{Q}+\frac{1}{3} \mathbf{I}\right)+2 \lambda(\nabla \mathbf{v}: \mathbf{Q})\left(\mathbf{Q}+\frac{1}{3} \mathbf{I}\right)\right],
\end{array}\right.
$$

where $\mathbf{D}$ is the rate of strain tensor, $p$ is the pressure, $\eta$ is the solvent viscosity, $\nabla \mathbf{v}$ is the velocity gradient, $n$ is the number of LCP molecules per unit volume, and $\lambda$ is the relaxation time of the LCP molecules associated with rotation of the dumbbell molecules. Note this constitutive equation accounts for a viscous stress as well as an LCP contribution [2].

Orientation tensor equation

$$
\left\{\begin{array}{l}
\frac{d}{d t} \mathbf{Q}-\left(\nabla \mathbf{v} \cdot \mathbf{Q}+\mathbf{Q} \cdot \nabla \mathbf{v}^{T}\right)=\frac{2}{3} \mathbf{D}-2(\nabla \mathbf{v}: \mathbf{Q})\left(\mathbf{Q}+\frac{1}{3} \mathbf{I}\right) \\
-\frac{\sigma}{\lambda}\left\{\left(1-\frac{N}{3}\right) \mathbf{Q}-N(\mathbf{Q} \cdot \mathbf{Q})+N(\mathbf{Q}: \mathbf{Q})\left(\mathbf{Q}+\frac{1}{3} \mathbf{I}\right)\right\}
\end{array}\right.
$$

where $0<\sigma \leq 1$ is the anisotropic drag coefficient measuring the hydrodynamic drag that a LCP molecule experiences as it moves relative to the solvent $(\sigma=1$ indicates the friction is isotropic, whereas $\sigma=0$ corresponds to the case where the friction is hyper-anisotropic so that the molecular motion is confined only to the direction of the molecular axis).

In [9], we introduced a biaxial representation for $\mathbf{Q}$ :

$$
\mathbf{Q}=s\left(\mathbf{n}_{3} \otimes \mathbf{n}_{3}-\frac{1}{3} \mathbf{I}\right)+\beta\left(\mathbf{n}_{2} \otimes \mathbf{n}_{2}-\frac{1}{3} \mathbf{I}\right),
$$

where $s$ and $\beta$ are two independent nematic LCP order parameters, equivalent to the eigenvalues of $\mathbf{Q}$, that carry nonlinear average information about the degree of orientation that the LCP molecule $\mathbf{m}$ makes with the orthonormal eigenvectors (so-called nematic directors) $\mathbf{n}_{i}, \mathrm{i}=1,2,3$, of $\mathbf{Q}$, defined by

$$
\left\{\begin{array}{l}
s=\left\langle\left(\mathbf{n}_{3} \cdot \mathbf{m}\right)^{2}\right\rangle-\left\langle\left(\mathbf{n}_{1} \cdot \mathbf{m}\right)^{2}\right\rangle \\
\beta=\left\langle\left(\mathbf{n}_{2} \cdot \mathbf{m}\right)^{2}\right\rangle-\left\langle\left(\mathbf{n}_{1} \cdot \mathbf{m}\right)^{2}\right\rangle .
\end{array}\right.
$$

The range of values for $(s, \beta)$ is the closed triangular region in the $(s, \beta)$ plane with vertices $(1,0),(0,1)$ and $(-1,-1)$.

Using this representation, we easily find several families of constant solutions for the complete system of governing equations, exhibiting uniaxial symmetry with respect to an arbitrary uniaxial nematic director $\mathbf{n}_{3}$,

$$
\mathbf{v}=\mathbf{v}_{0}, p=p_{0}, \mathbf{Q}=s\left(\mathbf{n}_{3} \mathbf{n}_{3}-\frac{\mathbf{I}}{3}\right)
$$

where $\mathbf{v}_{\mathbf{0}}$ is a constant vector, $p_{0}$ is a scalar constant pressure, $s$ is an extremum of the uniaxial bulk free energy $\int U(s) d s$, given by the zeros of

$$
U(s)=s\left(1-\frac{1}{3} N(1-s)(2 s+1)\right) .
$$

When $0<s \leq 1$, the liquid crystal is said to exhibit prolate uniaxial symmetry; when $-\frac{1}{2} \leq s<0$, one infers oblate uniaxial symmetry; $s=0$ corresponds to an isotropic state. 
Both the isotropic phase and the oblate uniaxial phase have recently been described as defects, based on the defining property that the major director is not uniquely defined in either phase [18]. The variation of the uniaxial order parameter equilibria as functions of the polymer concentration and their orientational stability properties absent of flows or in a constant velocity field is depicted in Figure 1, which has appeared in numerous papers. (We remark that if one arbitrarily restricts to the uniaxial order parameter dynamics, the oblate phase is mischaracterized as stable; indeed, this phase is always 2-dimensionally unstable due purely to orientational dynamics, independent of flow [7].)

\section{Remark:}

The uniaxial director $\mathbf{n}_{3}$, due to the absence of an inhomogeneous flow direction or an external body force, is arbitrary and can be parametrized by two angle parameters $\psi, \chi$ :

$$
\mathbf{n}_{3}=(\sin (\psi) \cos (\chi), \sin (\psi) \sin (\chi), \cos (\psi))=\mathbf{e}_{3} \mathbf{O}_{2} \mathbf{O}_{1},
$$

where

$$
\begin{aligned}
& \mathbf{O}_{1}=\left(\begin{array}{ccc}
\cos (\chi) & \sin (\chi) & 0 \\
-\sin (\chi) & \cos (\chi) & 0 \\
0 & 0 & 1
\end{array}\right), \mathbf{O}_{2}=\left(\begin{array}{llr}
\cos (\psi) & 0 & \sin (\psi) \\
0 & 1 & 0 \\
-\sin (\psi) & 0 & \cos (\psi)
\end{array}\right) \\
& \mathbf{e}_{3}=(0,0,1)
\end{aligned}
$$

If we denote $\mathbf{O}=\left(\mathbf{O}_{2} \mathbf{O}_{1}\right)^{T}$, then,

$$
\mathbf{Q}=s\left(\mathbf{n}_{3} \mathbf{n}_{3}-\frac{\mathbf{I}}{3}\right)=\mathbf{O} \mathbf{Q}_{0} \mathbf{O}^{T}, \mathbf{Q}_{0}=s\left(\mathbf{e}_{3} \mathbf{e}_{3}-\frac{\mathbf{I}}{3}\right) \text {. }
$$

Since $\mathbf{n}_{3}$ can be transformed into $\mathbf{e}_{3}$ through the rotation matrix $\mathbf{O}$, the conclusions obtained in this study with respect to director $\mathbf{e}_{3}$ apply equally to the case of arbitrary $\mathbf{n}_{3}$. Thus, we will only present our results for $\mathbf{n}_{3}=\mathbf{e}_{3}$ in the following.

The focus of the analysis to follow is the ill-posed, and therefore unphysical, behavior of the linearized equations in certain equilibria. For this reason, we simplify the presentation by assuming the density $\rho=1$ and by choosing unity as the characteristic time $\left(t_{0}\right)$ and length $\left(L_{0}\right)$ scale. Indeed there is no basic velocity scale to select in the nondimensional equations to follow, so we simply set the scales to unity. From here on all expressions are given in dimensionless form.

\section{The Doi model without solvent viscosity: $\eta=0$}

\subsection{Isotropic phases}

First we consider the linearized behavior of the original Doi model equations for an isotropic orientation equilibrium. The following normal modes are deduced for the constantcoefficient, linearized equations:

$$
(\bullet)(x, y, z, t)=e^{c t-i(k x+m y+n z)}(\tilde{\bullet})
$$


where $(\bullet)(x, y, z, t)$ is any one of the perturbed variables $\mathbf{v}, p, \mathbf{Q},(\tilde{\bullet})$ corresponds to the constant amplitude, $\mathrm{c}$ is the (possibly complex) wave speed, and $\mathbf{k}=(k, m, n)$ is the wavevector of wave numbers in the three coordinate directions, respectively. For simplicity, we let $\mathbf{v}_{0}=(0,0,0)$ since the result holds for any constant velocity. The linearized dispersion relation consists of all solution branches for the wave speed $c$ as a function of the wavevector k. We find seven wave speed branches, five from the orientation tensor degrees of freedom and two from the velocity field. These seven wave speeds yield a combination of linearized growth rates (if the real part of the wave speeds is positive), decay rates (if the real part of the wave speeds is negative), or neutral rates otherwise. These seven branches can be explicitly calculated, and are given by

$$
\begin{aligned}
& c_{1}=c_{2}=c_{3}=-\frac{\sigma}{\lambda}\left(1-\frac{1}{3} N\right), \\
& c_{4}=c_{5}=\frac{1}{2}\left[c_{1}+\sqrt{\phi}\right], \\
& c_{6}=c_{7}=\frac{1}{2}\left[c_{1}-\sqrt{\phi}\right], \\
& \phi=\left(c_{1}\right)^{2}-4 \alpha\left(1-\frac{1}{3} N\right)\left(|\mathbf{k}|^{2}\right),
\end{aligned}
$$

where $\alpha=\frac{3 n B T}{\rho\left(L_{0} / t_{0}\right)^{2}}$ is a dimensionless measure of the polymeric stress.

First we recall from [7] that the Doi orientation equation (6) absent of flow, with $\mathbf{v}=\mathbf{0}$, and in the isotropic equilibrium phase, has five identical linearized wave speeds: $c_{i}=-\frac{\sigma}{\lambda}(1-$ $\left.\frac{1}{3} N\right), i=1, \ldots, 5$. From this result it is apparent that the isotropic phase is either fivedimensionally stable $(N<3)$ or unstable $(N>3)$ in the absence of a flow field. The above wave speeds resolve the flow corrections to the orientation rates as well as the flow-dominated rates and their orientation corrections.

The first three wave speeds, $c_{1,2,3}$, are identical to the pure orientation rates, reflecting that near flow equilibrium there is no linear coupling to these orientation modes. We note that the flow is the only source of wavevector-dependent wave speeds. The remaining wave speeds, $c_{4,5,6,7}$, capture the linearized hydrodynamic-orientation interaction; $c_{4,5}$ resolve the flow coupling to the orientation rates, while $c_{6,7}$ limit to the zero linearized rates of the pure inviscid fluid equations.

- Case 1: Low LCP concentration.

When $N<3$, all wave speeds are negative, and the low-density isotropic equilibrium is stable in the nematic and fluid dynamics.

- Case 2: Transition concentration.

When $N=3$, the linearized constitutive equation reduces to that for inviscid fluids (the Euler equations) so that all modes become neutrally stable.

- Case 3: High LCP concentration.

When $N>3$, the three pure orientation wave speeds, $c_{1,2,3}$, yield constant unstable growth rates. In addition, $c_{4,5}$ provide two more enhanced, wavevector-dependent growth rates, which equal $c_{1}$ for a zero wavevector perturbation, and then grow proportional to $|\mathbf{k}|$. 
This is referred to as catastrophic, or Hadamard, instability, since the growth rates are unbounded in the small scale limit as $|\mathbf{k}|$ goes to infinity, growing linearly with $|\mathbf{k}|$. Such behavior occurs for an elliptic linear equation (e.g., Laplace's equation) when it is posed as an initial value problem, treating a space-like variable as a time-like variable. The growth rates $c_{4,5}$ imply that arbitrarily small lengthscale perturbations from mechanical and orientation equilibrium grow arbitrarily fast, a clearly unphysical prediction. The remaining two wave speeds, $c_{6,7}$, are zero for $\mathbf{k}=\mathbf{0}$, and then yield decay rates proportional to $|\mathbf{k}|$.

Thus, the isotropic phase has a five-dimensional instability for all nonzero wavevector perturbations of velocity, pressure and the orientation tensor. This unstable dimension is the same as the pure orientation dynamics, except that the two flow-driven growth rates, $c_{4,5}$, are unbounded in the small scale limit.

We note that this ill-posed behavior resides in the coupling of orientation and hydrodynamics; the inviscid Euler equations are Hamiltonian and neutrally stable at flow equilibrium, and the flow-independent Doi model is spatially homogeneous with constant linearized eigenvalues, independent of spatial wavevector. Furthermore, previous analyses of the same Doi flow/orientation equations with a non-trivial, imposed elongational or shear flow kinematics $[17,18,16,14,3,7]$ yield no such catastrophic behavior. In those calculations, there is no allowance for spatially varying flow perturbations which allow the nematic instabilities to couple to spatial structure.

This ill-posedness can also be interpreted in another way. Suppose we posit an "effective linearized Doi model for the isotropic phase", consisting of the following LCP constitutive relation and orientation equation for the isotropic phase:

$$
\begin{aligned}
& \hat{\tau}=3 n B T\left(1-\frac{N}{3}\right) \mathbf{Q}, \\
& \frac{d}{d t} \mathbf{Q}-\left(\nabla \mathbf{v} \cdot \mathbf{Q}+\mathbf{Q} \cdot \nabla \mathbf{v}^{T}\right)=-\frac{\sigma}{\lambda}\left(1-\frac{N}{3}\right) \mathbf{Q}+\frac{2}{3} \mathbf{D} .
\end{aligned}
$$

This effective linearized Doi model retains precisely those terms from the original Doi model which contribute to the linearized dynamics. Thus, this model shares the same constant flow equilibria and isotropic orientation steady state, and the exact same linearized dispersion relations.

If we now make the linear change of variables from $\mathbf{Q}$ to $\tau$, then the effective linearized Doi equation (16) is precisely identified with the upper-convected Maxwell model for isotropic viscoelastic fluids:

$$
\lambda_{e f f}\left[\frac{d}{d t} \hat{\tau}-\left(\nabla \mathbf{v} \cdot \hat{\tau}+\hat{\tau} \cdot \nabla \mathbf{v}^{T}\right)\right]+\hat{\tau}=2 \eta_{e f f} \mathbf{D}
$$

where $\eta_{\text {eff }}=n B T \frac{\lambda}{\sigma}$ is an effective polymer viscosity and $\lambda_{\text {eff }}=\frac{\lambda}{\sigma\left(1-\frac{1}{3} N\right)}$ is an effective polymer relaxation time.

This correspondence is valuable in understanding why the isotropic orientation phase experiences pathological behavior at high LCP concentration. When $N<3$, the effective relaxation time is positive, and the linearized behavior is identical to the Maxwell fluid near equilibrium; the equations are locally well-posed in the neighborhood of a zero extra-stress tensor [11]. When $N>3$, the effective relaxation time, $\lambda_{\text {eff }}$, is negative, and therefore the 
linearized model becomes nonphysical with the nearly zero extra stress undergoing amplification instead of relaxation.

\section{Commentary.}

Since this effective model has the same linearized behavior as the original Doi model near isotropic equilibrium, we conclude the models are unphysical at high concentrations and near the isotropic state. We have struggled without success to physically rationalize this behavior, although there may be some physical explanation as to how anisotropic microstructure, in an unstable phase that wants to go nematic, can be driven by the flow interaction to a linearized dynamics that is violently unstable. Therefore other physical effects, ignored on the basis of being relatively weak, have to be accounted for to arrest the behavior. This behavior has precedent in the Rayleigh-Taylor instability, which is linearly ill-posed; thus any numerical or theoretical predictions of the local dynamics during departure from equilibrium require the addition of regularizing physical effects, surface tension in the Rayleigh-Taylor case. We shall return to this point of view in Section 4 after we document the linearized dynamics of anisotropic/flow steady states.

\subsection{Anisotropic nematic phases}

Now we examine the linearized Doi model in a neighborhood of an anisotropic nematic equilibrium $s_{e q}$, where $s_{e q}$ is a root of $\frac{U(s)}{s}=0$. Recall that nematic phases occur only for high concentrations, $N \geq \frac{8}{3}$. Also recall that we have arbitrarily selected the uniaxial nematic director axis to be aligned with the z-axis, and that the perturbed wavevector has rectangular coordinates $(k, m, n)$, so that the z-axis wavenumber $n$ is aligned with the director axis.

The linearized dispersion equation yields seven wave speeds. We caution that an explicit ordering of the branches of the dispersion relation, that identifies the first five $c_{i}$ with pure orientation wave speeds and the last two $c_{i}$ with fluid wave speeds, is very difficult. Rather, we will list the formulae and then account for all five orientation and two fluid wave speeds in the decoupled limit as $\mathbf{k}$ approaches zero.

Two wave speeds for anisotropic equilibria are identically zero,

$$
c_{1}=c_{2}=0
$$

these zero linearized eigenvalues correspond to the two arbitrary phase symmetries of the uniaxial nematic director $\mathbf{n}_{3}$. Two more wave speeds have a compact expression,

$$
\begin{aligned}
& c_{3}=-\frac{1}{2}\left[\frac{N \sigma}{\lambda} s_{e q}+\sqrt{\Pi}\right], \\
& c_{4}=-\frac{1}{2}\left[\frac{N \sigma}{\lambda} s_{e q}-\sqrt{\Pi}\right], \\
& \Pi=\left(\frac{N \sigma}{\lambda} s_{e q}\right)^{2}+\frac{4}{3} \alpha s_{e q}\left(s_{e q}-1\right)\left(m^{2}+k^{2}\right) N .
\end{aligned}
$$

The remaining three wave speeds, $c_{5,6,7}$, are roots of a cubic equation, for which we give the asymptotic formulae for both long and short wavelength perturbations since any other 
representation of the roots is unenlightening:

Leading order asymptotic formulae for short wavelengths $(|\mathbf{k}|>>1)$

$$
\begin{aligned}
& c_{5} \sim-\frac{2}{|\mathbf{k}|^{2}} \alpha \lambda s_{e q}^{2} n^{2}\left(k^{2}+m^{2}\right), \\
& c_{6} \sim-\frac{N}{6 \lambda}\left[\left(1+4 s_{e q}\right)\left(1-s_{e q}\right)+2 \sigma s_{e q}\left(2 s_{e q}+1\right)-\left(1-s_{e q}\right) \sqrt{\Xi}\right], \\
& c_{7} \sim-\frac{N}{6 \lambda}\left[\left(1+4 s_{e q}\right)\left(1-s_{e q}\right)+2 \sigma s_{e q}\left(2 s_{e q}+1\right)+\left(1-s_{e q}\right) \sqrt{\Xi}\right], \\
& \Xi=\left(4 s_{e q}+1\right)^{2}+8 \sigma\left(1-s_{e q}\right)+16 \sigma s_{e q}^{2}(\sigma-2) .
\end{aligned}
$$

Leading order asymptotic formulae for long wavelengths $(|\mathbf{k}|<<1)$

$$
\begin{aligned}
& c_{5} \sim-\frac{n^{2}\left(k^{2}+m^{2}\right)}{|\mathbf{k}|^{2}} \frac{\alpha \lambda}{3 \sigma}\left[2+s_{e q}-3(1-\sigma) s_{e q}^{2}\right], \\
& c_{6} \sim-\frac{\sigma N}{\lambda} s_{e q}, \\
& c_{7} \sim-\frac{\sigma}{\lambda} U^{\prime}\left(s_{e q}\right) .
\end{aligned}
$$

Remarks:

(i). The labelling of $c_{5}, c_{6}$ and $c_{7}$ in (20) and (21) is arbitrary, as the roots of the cubic are not uniquely ordered as one goes from one limit to the other.

(ii). We now confirm that these wave speed formulas limit to the exact linearized dynamics when the orientation and flow are decoupled. Absent of flow, the five linearized eigenvalues $\mu_{i}, i=1, \ldots, 5$ of the pure orientation dynamics for a uniaxial, anisotropic, nematic equilibrium are given in [7]:

$$
\begin{aligned}
& \mu_{1}=\mu_{2}=0, \\
& \mu_{3}=\mu_{4}=-N \frac{\sigma}{\lambda} s_{e q}, \\
& \mu_{5}=-\frac{\sigma}{\lambda} U^{\prime}\left(s_{e q}\right) .
\end{aligned}
$$

Thus, a comparison with the flow/orientation wave speeds in the limit as $\mathbf{k}$ goes to zero yields the correspondence: $c_{1,2}$ are identified with $\mu_{1,2} ; c_{4,6}$ are identified with $\mu_{3,4}$; and $c_{7}$ is identified with $\mu_{5}$; finally, $c_{3,5}$ correspond to the neutral linearized behavior of the inviscid fluid equations near equilibrium. The formulas given here can now be confidently interpreted to represent the coupled linearized dynamics of flow and anisotropic nematic structure.

- (i) Highly aligned prolate equilibria $\left(s_{e q} \geq \frac{1}{4}\right)$

When $s_{e q} \geq \frac{1}{4}$, there are only neutral wave speeds $\left(c_{1,2}\right)$ and decay rates $\left(c_{3,4,5,6,7}\right)$ : the highly aligned prolate equilibrium is neutrally stable. As noted above, the two zero linearized eigenvalues correspond to the two arbitrary angle degrees of freedom of the nematic director axis-there is no distinguished flow or nematic direction in this problem, so the nematic director can lie anywhere on the unit sphere. 
- (ii) Less aligned prolate equilibria $\left(0<s_{\text {eq }} \leq \frac{1}{4}\right)$

When $0<s_{e q}<\frac{1}{4}$, which occurs for $\frac{8}{3}<N<3$, it is easy to see that $U^{\prime}\left(s_{e q}\right)<0$, so that $\operatorname{Re}\left(c_{7}\right)$ is positive in the long wavelength range. It can then be confirmed that $\operatorname{Re}\left(c_{7}\right)>0$ and bounded above for all wavevectors $\mathbf{k}$ (note that the positive growthrate is labeled $c_{6}$ for $|\mathbf{k}|>>1$ ). Therefore, this equilibrium is unstable with a one-dimensional instability; since the unstable growthrate is bounded, the linearized equations are well-posed.

- (iii) Oblate equilibria $\left(-\frac{1}{2} \leq s_{e q}<0\right)$

When $s_{e q}<0$, which exists for $N>3$, the flow/nematic equilibrium is always unstable, but the dimension of instability and boundedness of the growthrates depend on the wavevector; i.e., the instability of the oblate nematic phase is itself spatially anisotropic, or "directional", by analogy with directional solidification.

Observe first that $c_{6}$ is positive in the long wavelength limit, and then one can check that it remains positive and bounded for all wavevectors. Thus the instability is at least one-dimensional in all wavevector perturbations, and $c_{6}$ does not contribute to ill-posed behavior.

The wave speed $c_{3}$ is associated with the neutral rate of the inviscid fluid equations, and limits to zero for $\mathbf{k}=\mathbf{0}$. Remarkably, $c_{3}=0$ if $k=m=0$, i.e., if the perturbation wavevector is either parallel to the z-axis or homogeneous, and $c_{3}<0$ (corresponding to a stable decay rate) if and only if the perturbation wavevector has nonzero projection orthogonal to the nematic director axis (the z-axis), i.e., if and only if $k m \neq 0$. This particular wave speed thus undergoes a directional stability corresponding to the microstructural influence on the inviscid neutral linearized dynamics.

The wave speed $c_{4}$ yields linearized instability for all wavevectors, but with dramatic dependence on the wavevector! If $\mathbf{k} \neq \mathbf{0}$ is not parallel to the uniaxial nematic director axis (z-axis), i.e., $k$ or $m \neq 0$, then the growth rate is proportional to $\sqrt{k^{2}+m^{2}}$, which is unbounded in the small wavelength limit. Thus we deduce Hadamard-type catastrophic instability for perturbation wavevectors non-parallel to $\mathbf{n}_{3}$. If the perturbation wavevector is parallel to the director axis, however, then $c_{4}$ limits to the constant, pure orientation growthrate $\mu_{3},(22)$.

We also comment that although polymeric rotary diffusion is present in this model, as exhibited through $c_{5}$, it is not strong enough to suppress the behavior deduced here. Coincidentally, both incidents of Hadamard instability are deduced in equilibria associated with defects, where nematic isotropy dominates in the directions of unbounded growth. These results suggest to us that if one were able somehow to prepare the unstable isotropic or oblate nematic phases, above critical LCP density, then the isotropic directions of the nematic in the presence of a weak flow will very rapidly depart from isotropic phases, presumably toward the neutrally stable prolate equilibrium. 


\section{Regularizing effects of solvent viscosity, long-range intermolecular potentials, and spatial inhomogeneity}

We now investigate how this catastrophic behavior is regularized by accounting for natural physical effects that are present in LCP flows, though they may be quite weak: solvent viscosity, long-range interactions in excluded-volume potentials, and spatial inhomogeneity in LCP concentration. Each of these effects introduces spatial gradients (of $\mathbf{v}, \mathbf{Q}$, and $N$, respectively), which have the potential to arrest the ill-posed behavior of the high-density isotropic and oblate nematic phases in weak flows.

\subsection{Regularization by solvent viscosity}

First we consider solvent viscosity in the constitutive equation (5), with $\eta \neq 0$.

\subsubsection{Isotropic phases}

The seven wave speeds for isotropic equilibria, $s_{e q}=0$, become

$$
\begin{aligned}
& c_{1}=c_{2}=c_{3}=-\frac{\sigma}{\lambda}\left(1-\frac{1}{3} N\right), \\
& c_{4}=c_{5}=-\frac{1}{2}\left[\frac{1}{R e}|\mathbf{k}|^{2}+\frac{\sigma}{\lambda}\left(1-\frac{1}{3} N\right)-\sqrt{\phi}\right], \\
& c_{6}=c_{7}=-\frac{1}{2}\left[\frac{1}{R e}|\mathbf{k}|^{2}+\frac{\sigma}{\lambda}\left(1-\frac{1}{3} N\right)+\sqrt{\phi}\right], \\
& \phi=\left[\frac{1}{R e}|\mathbf{k}|^{2}+\frac{\sigma}{\lambda}\left(1-\frac{1}{3} N\right)\right]^{2}-4\left(1-\frac{1}{3} N\right)\left(\frac{\sigma}{R e \lambda}+\alpha|\mathbf{k}|^{2}\right),
\end{aligned}
$$

where $R e=\frac{\rho L_{0}^{2}}{\eta t_{0}}$ is the solvent Reynolds number, and the length scale $L_{0}$ and time scale $t_{0}$ have been set to unity.

Once again we note that in the limit $\mathbf{k}=\mathbf{0}$ and $\frac{1}{R e}=0$, we reproduce the five pure orientation and two inviscid linearized rates. The addition of solvent viscosity does not significantly alter the long wavelength behavior of the unstable growth rates presented in the previous section, but bounds the growth rates associated to short wavelength perturbations, with no finite wavelength cutoff in the unstable wavevectors. At $N=3$, the result is Newtonian, i.e., orientation information is lost and decouples from the hydrodynamics, which is exhibited through five neutral orientation rates and two viscous fluid decay rates.

\subsubsection{Anisotropic phases}

The seven wave speeds for anisotropic equilibria consist of four explicit formulas and three 
which can only be provided in asymptotic short and long wavelength form:

$$
\begin{aligned}
& c_{1}=c_{2}=0, \\
& c_{3}=-\frac{1}{2}\left[\frac{1}{R e}|\mathbf{k}|^{2}+\frac{N \sigma}{\lambda} s_{e q}+\sqrt{\Pi}\right], \\
& c_{4}=-\frac{1}{2}\left[\frac{1}{R e}|\mathbf{k}|^{2}+\frac{N \sigma}{\lambda} s_{e q}-\sqrt{\Pi}\right], \\
& \Pi=\left[\frac{1}{R e}|\mathbf{k}|^{2}-\frac{N \sigma}{\lambda} s_{e q}\right]^{2}+\frac{4}{3} N \alpha s_{e q}\left(s_{e q}-1\right)\left(m^{2}+k^{2}\right) .
\end{aligned}
$$

Remaining wave speeds for short wavelengths $(|\mathbf{k}|>>1)$

$$
\begin{aligned}
& c_{5} \sim-\frac{1}{R e}|\mathbf{k}|^{2}-2 \alpha \lambda s_{e q}^{2} \frac{n^{2}\left(k^{2}+m^{2}\right)}{|\mathbf{k}|^{2}}, \\
& c_{6} \sim \frac{-b+\sqrt{b^{2}-4 a c}}{2 a}, \\
& c_{7} \sim \frac{-b-\sqrt{b^{2}-4 a c}}{2 a}, \\
& a=\frac{1}{R e}|\mathbf{k}|^{4}+2 \alpha \lambda s_{e q}^{2} n^{2}\left(k^{2}+m^{2}\right), \\
& b=\frac{2 N s_{e q}}{3}\left[\frac{1}{R e} \frac{\sigma}{\lambda}\left(|\mathbf{k}|^{2}\right)^{2}\left(1+2 s_{e q}\right)+\alpha n^{2}\left(k^{2}+m^{2}\right) s_{e q}\left(\left(1+4 s_{e q}\right)\left(1-s_{e q}\right)+2 \sigma s_{e q}\left(1+2 s_{e q}\right)\right)\right], \\
& c=-\frac{\sigma N^{2} s_{e q}^{2}}{9 \lambda}\left[\frac{3 \sigma}{R e \lambda}\left(1-4 s_{e q}\right)|\mathbf{k}|^{4}+\alpha n^{2}\left(k^{2}+m^{2}\right)\left(1-4 s_{e q}\right)\left(\left(2\left(1-s_{e q}\right)\left(3 s_{e q}+2\right)+6 \sigma s_{e q}^{2}\right)\right)\right] .
\end{aligned}
$$

Remaining wave speeds for long wavelengths $(|\mathbf{k}|<<1)$

$$
\begin{aligned}
& c_{7} \sim-\frac{1}{|\mathbf{k}|^{2}}\left[\frac{1}{R e}|\mathbf{k}|^{4}+\frac{\alpha \lambda}{3 \sigma}\left(2+s_{e q}-3(1-\sigma) s_{e q}^{2}\right) n^{2}\left(k^{2}+m^{2}\right)\right], \\
& c_{5} \sim-\frac{\sigma N}{\lambda} \\
& c_{6} \sim-\frac{\sigma}{\lambda} U^{\prime}\left(s_{e q}\right) .
\end{aligned}
$$

- (i) Highly aligned prolate equilibria $\left(s_{e q} \geq \frac{1}{4}\right)$

All seven wave speeds yield decay rates: the highly aligned prolate uniaxial nematic phases, parametrized by LCP concentration $N>\frac{8}{3}$, at flow equilibrium are linearly stable.

- (ii) Less aligned prolate equilibria $\left(0<s_{\text {eq }} \leq \frac{1}{4}\right)$

When $0<s_{e q}<\frac{1}{4}$, or equivalently for $\frac{8}{3}<N<3, c_{6}$ is the only positive growth rate, which is bounded for all wavevector perturbations. Therefore, the less-aligned prolate uniaxial phase at flow equilibrium is one-dimensionally unstable in all wavevector perturbations, and linearly well-posed. This is the same dimension of instability that exists in the flow-independent pure orientation dynamics and in the flow without viscosity; it originates in the uniaxial order parameter dynamics [17]. This calculation shows the intermediate prolate nematic phase in weak flow does not experience flow-induced enhancement of the nematic instability. 
- (iii) Oblate equilibria $\left(-\frac{1}{2} \leq s_{e q}<0\right)$

For the oblate nematic phases, $s_{e q}<0$, which exist for $N>3$, the wave speed $c_{4}$ is positive in addition to $c_{6}$, and both are bounded for all wavevector perturbations. Thus, the oblate uniaxial phases in flow equilibrium are two-dimensionally unstable with well-posed linearized dynamics in the presence of solvent viscosity.

We therefore conclude that the inclusion of an arbitrary non-zero solvent viscosity in the constitutive equation regularizes but does not stabilize unstable nematic orientation/flow equilibria; all flow/orientation equilibria that were unstable remain unstable in all wavelengths, with the single important consequence that solvent viscosity bounds all growth rates of all equilibria. This complete analysis of all flow/nematic equilibria establishes: the Doi flow/orientation model with solvent viscosity is linearly well-posed as an evolutionary (dynamical) system. In this sense, we say that solvent viscosity regularizes the original Doi model. We emphasize that solvent viscosity does not suppress short wavelength instabilities, rather it only bounds their growth in time. The growth rates and decay rates for the two unstable uniaxial equilibria are depicted in Figure 2 for $\frac{1}{R e}=0.5$.

\subsection{Regularization by finite-range (nonlocal) interaction in the excluded-volume potential}

Besides a solvent viscosity, we now show the nonlocal diffusive effect obtained by incorporating a finite-range effect in the intermolecular potential also regularizes the original Doi model. We consider the equation for inhomogeneous LCPs given by Bhave [1] with the Marrucci-Greco potential of finite-range interaction [13],

$$
V_{m-c}(\mathbf{m})=-\frac{3 N T}{2}\left(\mathbf{Q}+\frac{l^{2}}{24} \Delta \mathbf{Q}\right): \mathbf{m m},
$$

where $l$ measures the finite lengthscale of interaction. The constitutive and orientation tensor equations are modified to account for the new intermolecular potential. To save space, we refer to $[1,2]$ for details of the equations. Intuitively, we comment that this Laplacian of the orientation tensor is analogous to the Laplacian of the velocity field in Newtonian viscous fluids; we therefore anticipate a much stronger regularizing effect from this finiterange molecular interaction, with a strong quadratic "- $|\mathbf{k}|^{2}$ " decay in the linearized nearequilibrium solutions.

Note that the generalized governing system admits the same flow/nematic equilibria given at the end of section 2. We then repeat the linearized analysis.

\subsubsection{Isotropic equilibria}


The wave speeds for isotropic equilibria are

$$
\begin{aligned}
& c_{1}=c_{2}=c_{3}=-\frac{\sigma}{\lambda}\left(1-\frac{N}{3}\right), \\
& \left.c_{4}=c_{5}=-\frac{1}{2}\left[\frac{\sigma}{\lambda}\left(1-\frac{1}{3} N\right)+\left(\frac{1}{R e}+\frac{N l^{2}}{72}\right)|\mathbf{k}|^{2}\right]-\sqrt{\Delta}\right), \\
& \left.c_{6}=c_{7}=-\frac{1}{2}\left[\frac{\sigma}{\lambda}\left(1-\frac{1}{3} N\right)+\left(\frac{1}{R e}+\frac{N l^{2}}{72}\right)|\mathbf{k}|^{2}\right]+\sqrt{\Delta}\right), \\
& \Delta=\left[\frac{\sigma}{\lambda}\left(1-\frac{1}{3} N\right)+\left(\frac{1}{R e}+\frac{N l^{2}}{72}\right)|\mathbf{k}|^{2}\right]^{2}-\frac{4 \alpha}{3}\left[\left(1-\frac{1}{3} N\right)+\right. \\
& \left.\frac{N l^{2}}{72}|\mathbf{k}|^{2}\right]|\mathbf{k}|^{2}-\frac{4}{R e}\left[\frac{\sigma}{\lambda}\left(1-\frac{1}{3} N\right)+\frac{N l^{2}}{72}|\mathbf{k}|^{2}\right] .
\end{aligned}
$$

As in the previous cases, the first three wave speeds are purely orientational. They contribute three decay rates, neutral rates and growth rates for $N<3, N=3$ and $N>3$, respectively. The other four wave speeds, reflecting the hydrodynamic-orientation coupling, all yield decay rates in the range of short wavelengths. For long wavelengths, there are two growth rates for $N>3$. This scenario persists regardless of the value of the Reynolds number, i.e., independent of solvent viscosity. Therefore, for these wavevector-dependent modes, the effect of a nonlocal, finite-range intermolecular interaction is to regularize the linearized dynamics and stabilize all short wavelength perturbations.

This regularization is distinguished from the two previous Doi models in that all unstable growth rates now have a finite wavelength cutoff, analogous to the viscous fluid equations near flow equilibrium. This property of (28) holds with or without solvent viscosity.

\subsubsection{Anisotropic equilibria}

We simply note here that for anisotropic equilibria the primary conclusions obtained in the above isotropic case again hold true: the spatially-dependent linearized growth rates are both reduced in magnitude and suppressed beyond a finite wavelength cutoff. We do not give the details here; the authors may be contacted for the cumbersome formulae.

\subsection{Regularization by spatial inhomogeneity}

When spatial diffusion is considered in the Doi theory, the linearized dynamics is also regularized in a sense to be discussed next. In this generalized theory, the polymer concentration at each spatial location and time is variable and dynamically interacts with the other effects. Thus a new equation must be derived for this interaction. We will sketch the result of incorporating this effect.

Let $\gamma$ denote the polymer concentration variable, which is identified with the parameter $N$ for spatially homogeneous LCPs. The time evolution equation of $\gamma$ is obtained by averaging within the framework of the Doi kinetic theory [1]:

$$
\begin{aligned}
& \frac{D \gamma}{D t}=D_{t r}\left\{\sigma \gamma_{, i i}+(1-\sigma)\left(\gamma \mathbf{M}_{i j}\right)_{, j i}-\frac{3}{2}\left[\sigma\left(\left(\gamma \mathbf{Q}_{m n}\right)_{, i}\left(\gamma \mathbf{M}_{m n}\right)\right)_{, i}+\right.\right. \\
& \left.\left.(1-\sigma)\left(\left(\gamma \mathbf{Q}_{m n}\right)_{, j} \gamma \mathbf{M}_{i j} \mathbf{M}_{m n}\right)_{, i}\right]+\frac{8}{5}\left[\sigma(\gamma, \gamma \gamma)_{, i}+(1-\sigma)\left(\gamma_{, j} \gamma \mathbf{M}_{i j}\right)_{, i}\right]\right\} .
\end{aligned}
$$

where $D_{t r}$ is the spatial diffusion coefficient, $\mathbf{M}=\mathbf{Q}+\frac{\mathbf{I}}{3}, \mathbf{Q}=\left\langle\mathbf{m m}-\frac{\mathbf{I}}{3}\right.$, the Einstein notation of tensor summation is used, and $(\bullet)_{, i}$ denotes the partial derivative with respect to $x_{i}$ in 
Cartesian coordinates $\left(x_{1}, x_{2}, x_{3}\right)$. Due to the variable concentration $\gamma$, the Maier-Saupe potential is generalized to

$$
V_{g m s}=-\frac{3}{2} \gamma B T\left(\mathbf{m m}-\frac{\mathbf{I}}{3}\right): \mathbf{Q}+\frac{8}{5} \gamma B T .
$$

The time evolution equation for $\mathbf{Q}$ then takes the form ([1]):

$$
\begin{aligned}
& \frac{D}{D t} \mathbf{Q}_{k l}-\left(\nabla \mathbf{v}_{k, i} \mathbf{Q}_{i, l}+\mathbf{Q}_{k i} \nabla \mathbf{v}_{l, i}\right)=\frac{D_{t r}}{\gamma}\left[\sigma\left(\gamma \mathbf{Q}_{k l}\right)_{, i i}+(1-\sigma)\left(\gamma \mathbf{M}_{i j} \mathbf{Q}_{k l}\right)_{, j i}-\right. \\
& \frac{3}{2}\left[\sigma\left(\left(\gamma \mathbf{Q}_{m n}\right)_{, i} \gamma \mathbf{M}_{m n} \mathbf{Q}_{k l}\right), i+(1-\sigma)\left(\left(\gamma \mathbf{Q}_{m n}\right)_{, j} \gamma \mathbf{M}_{i j} \mathbf{M}_{m n} \mathbf{Q}_{k l}\right)_{, i}\right]+ \\
& \frac{8}{5}\left[\sigma\left(\gamma\left(\gamma, i \mathbf{Q}_{k l}\right)_{, i}+(1-\sigma)\left(\gamma, j \mathbf{M}_{i j} \mathbf{Q}_{k l}\right)_{, i}\right]-\frac{\mathbf{Q}_{k l}}{\gamma} \frac{D \gamma}{D t}+\frac{2}{3} \mathbf{D}_{k l}-\right. \\
& \frac{\sigma}{\lambda}\left[\mathbf{Q}_{k l}-\frac{\gamma}{2}\left(\mathbf{Q}_{k i} \mathbf{M}_{i l}+\mathbf{M}_{k i} \mathbf{Q}_{i l}\right)+\gamma \mathbf{Q}_{m n} \mathbf{M}_{m n} \mathbf{M}_{k l}\right]-2 \mathbf{D}_{m n} \mathbf{M}_{m n} \mathbf{M}_{k l} .
\end{aligned}
$$

The extra stress equation becomes [1]:

$$
\begin{aligned}
& \hat{\tau}=2 \eta \mathbf{D}+3 a B T \gamma \mathbf{Q}+3 a \gamma^{2} B T[\mathbf{Q}: \mathbf{M}(\mathbf{M})- \\
& (\mathbf{M}) \mathbf{Q}]+6 a B T \lambda \gamma(\nabla \mathbf{v}: \mathbf{Q})(\mathbf{M}),
\end{aligned}
$$

where $a=\frac{8}{L^{2} b 5 \pi}, L$ is the length and $b$ is the radius of the rodlike molecule.

Remark:

The orientation tensor equation for $\mathbf{Q}$ is obtained from the second moment of $\mathbf{m}$ through

$$
\frac{D \mathbf{Q}}{D t}=\frac{1}{\gamma}\left[\frac{D}{D t}\left(\gamma\left\langle\mathbf{m m}-\frac{\mathbf{I}}{3}\right\rangle\right)-\mathbf{Q} \frac{D \gamma}{D t}\right],
$$

where the zero-trace condition is maintained. In addition to the quadratic closure, "cubic" closure approximation defined below is also used:

$$
\langle\mathbf{m m m m m m}\rangle=\langle\mathbf{m m}\rangle\langle\mathbf{m m}\rangle\langle\mathbf{m m}\rangle .
$$

For the modified governing equation of spatially inhomogeneous LCPs, a constant polymer concentration along with the equilibria given in section 2 constitute the equilibria for the modified system. Then, we repeat the linearized analysis on these equilibria.

\subsubsection{Isotropic equilibria}

For isotropic equilibria the wave speeds can be calculated explicitly. There are now eight wave speeds, with one additional wave speed due to the variable concentration:

$$
\begin{aligned}
& c_{1}=c_{2}=-\frac{1}{2}\left[\frac{\sigma}{\lambda}\left(1-\frac{\gamma}{3}\right)+\frac{D r}{3}(2 \sigma+1)\left(|\mathbf{k}|^{2}\right)+\frac{1}{R e}|\mathbf{k}|^{2}+\sqrt{\Delta}\right], \\
& c_{3}=c_{4}=-\frac{1}{2}\left[\frac{\sigma}{\lambda}\left(1-\frac{\gamma}{3}\right)+\frac{D r}{3}(2 \sigma+1)\left(|\mathbf{k}|^{2}\right)+\frac{1}{R e}|\mathbf{k}|^{2}-\sqrt{\Delta}\right], \\
& \Delta=\left[\frac{\sigma}{\lambda}\left(1-\frac{\gamma}{3}\right)+\frac{D r}{3}(2 \sigma+1)\left(|\mathbf{k}|^{2}\right)+\frac{1}{R e}|\mathbf{k}|^{2}\right]^{2}-\frac{4}{R e}|\mathbf{k}|^{2}\left[\frac{\sigma}{\lambda}\left(1-\frac{\gamma}{3}\right)+\right. \\
& \left.\frac{D r}{3}(2 \sigma+1)\left(|\mathbf{k}|^{2}\right)\right]-4 \frac{\alpha_{h}}{3} \gamma\left(1-\frac{\gamma}{3}\right)|\mathbf{k}|^{2} \text {, } \\
& c_{5}=c_{6}=c_{7}=-\frac{\sigma}{\lambda}\left(1-\frac{\gamma}{3}\right)-\frac{D r}{3}(2 \sigma+1)|\mathbf{k}|^{2}, \\
& c_{8}=-\frac{D r}{3}(1+2 \sigma)\left(1+\frac{8 \gamma}{5}\right)|\mathbf{k}|^{2},
\end{aligned}
$$


where $D r=\frac{D_{t r} t_{0}}{L_{0}^{2}}$ is the dimensionless diffusion coefficient and $\alpha_{h}=3 a B T$ measuring the strength of the orientation stress.

This newly introduced contribution due to the variable concentration lowers all wavevector rates, $\operatorname{Re}\left(c_{i}\right)$. The instability represented by $c_{5}, c_{6}, c_{7}$ in the long wavelength range is stabilized in the short wavelength range by the variable concentration. There are finite wavelength instability cutoff associated with these growthrates. However, the instability represented by $c_{3}$ and $c_{4}$, when $\gamma>3$, persists in short wavelength range if solvent viscosity is absent, i.e., $\frac{1}{R e}=0$, but there is a finite wavelength instability cutoff only if solvent viscosity is coupled to variable concentration. Therefore, the spatial diffusion effect regularizes the ill-posed Doi model, but does not necessarily stabilize the short wavelength instability.

\subsubsection{Anisotropic equilibria}

The dispersion equation for the anisotropic equilibria is an eighth-order polynomial in wave speed $c$. Since it is extremely long, we omit it here. Among the eight wave speeds, one is always analytically available:

$$
c_{1}=-\frac{D r}{3}\left[\left(\sigma\left(2 s_{e q}+1\right)+1-s_{e q}\right)\left(k^{2}+m^{2}\right)+\left(2 \sigma\left(1-s_{e q}\right)+2 s_{e q}+1\right) n^{2}\right] ;
$$

when $\sigma=1$, the second one is identically equal to the first one:

$$
c_{2}=c_{1} \text {. }
$$

The others must be calculated numerically. Even though we have their asymptotic formulae in the range of long and short wavelengths, respectively, we will not present them here due to their length. The results are available from the authors.

For the highly aligned prolate equilibrium, all wave speeds give decay rates, among which three are finite for the zero wavevector and others vanish. For the less aligned prolate equilibrium in the range $\frac{8}{3}<N<3$, there exists only one growth rate for finite wave numbers and all wave speeds yield decay rates for sufficiently large wavenumbers. The oblate equilibrium sustains two growth rates for sufficiently small wave numbers. As wavenumbers increase, one growth rate becomes a decay rate and one growth rate persists. If solvent viscosity is included as well, all growth rates vanish for high enough $\mathbf{k}$, and there is a finite wavenumber cutoff of the instability. A representative portrait for the growth/decay rates in the oblate and less aligned prolate equilibrium is given in Figure 2 and Figure 3, respectively.

\section{Conclusion}

We have solved the linearized Doi nematodynamic models near flow and nematic equilibria, and made explicit comparisons with the pure orientation and pure hydrodynamic linearized dynamics. From this analysis, we have deduced the consequences of hydrodynamic and orientation interactions, according to Doi-like models, for flows of LCPs in a neighborhood of flow/nematic equilibria. The key aspect of our analysis is an explicit characterization of the microstructure-flow coupling, which is analytically possible near these equilibria. The growth and decay rates of the orientation tensor dynamics, either with no flow or in the presence 
of an imposed simple shear or elongational kinematics, are constant independent of spatial wavenumbers. Here we allow the linearized fluid velocity and orientation tensor variables to depend on space coordinates, and then calculate the linearized dispersion relation for the full system of flow-orientation equations. Several growth and decay rates are wavevectordependent, the details of which are the essence of this paper.

We benchmark the analysis by recognizing that the flow and orientation dynamics decouples in the zero wavevector limit, and then confirming that all linearized wave speeds reduce to the pure orientation and pure fluid linearized rates in the limit of zero wavevector. The hydrodynamic-orientation coupling therefore has the greatest influence on the linearized dynamics in the intermediate and short wavelengths. As a general result, we find the uniaxial isotropic (low density) and nematic prolate (high density) equilibria that are stable absent of flow (Figure 1), remain stable in the coupled equations near mechanical equilibrium. Moreover, unstable isotropic (high density), nematic less-aligned prolate (intermediate density) and oblate (high density) phases remain unstable; however, the qualitative effect of the flow-orientation coupling depends critically on the physics included in the theory.

When rotary diffusion is the only diffusive effect in the theory,

- the less-aligned prolate equilibrium remains one-dimensionally unstable with a wavevectordependent but bounded growth rate;

- the isotropic equilibria for $N>3$ are five-dimensionally, unstable with two growth rates proportional to $|\mathbf{k}|$; and

- the oblate equilibria are two-dimensionally unstable with one growthrate proportional to $|(k, m, 0)|$, the directional wavevector transverse to the director axis $(0,0,1)$.

Hence, so-called Hadamard or catastrophic instability occurs for the original Doi model for equilibria in the unstable nematic phases which have been ascribed to nematic defects [13, 18]. By incorporating additional physical effects in the Doi theory, such as solvent viscosity, finite-range interaction in the excluded volume potential, and spatial inhomogeneity, we show the Hadamard instability is regularized. We note that the regularization effects are most significant in the short lengthscales, which is where the ill-posedness arises in the original Doi model.

Specifically, when a solvent viscosity is included in the Doi theory:

- all linearized growth, decay and neutral rates are reduced;

- growth rates in the high-density isotropic, less aligned prolate and oblate nematic equilibria persist, but are bounded independent of $\mathbf{k}$ so that Hadamard instability is suppressed.

When a finite-range intermolecular potential is coupled to the Doi theory,

- all unstable linearized modes have a finite wavevector unstable cutoff, so that all instabilities are in long to intermediate wavelength perturbations.

Finally, when spatial inhomogeneity is incorporated into the Doi model, 
- all linearized modes are stable to short wavelength perturbations except for the oblate and the high-density isotropic equilibria, which are unstable to all $\mathbf{k}$ with a bounded growth rate;

- when solvent viscosity is coupled along with spatial inhomogeneity, the short wavelength perturbations are stabilized for all equilibria.

\section{Acknowledgement and Disclaimer}

Effort sponsored by the Air Force Office of Scientific Research, Air Force Materials Command, USAF, under grant number F49620-96-1-0131 and F49620-97-1-0001. The US Government is authorized to reproduce and distribute reprints for governmental purposes notwithstanding any copyright notation thereon. The views and conclusions contained herein are those of the authors and should not be interpreted as necessarily representing the official policies or endorsements, either expressed or implied, of the Air Force Office of Scientific Research or the US Government.

\section{References}

[1] A. V. Bhave, "Kinetic theory for dilute and concentrated polymer solutions: study of nonhomogeneous effects", Ph. D. Thesis, MIT, 1992.

[2] A. V. Bhave, R. K. Menon, R. C. Armstrong, and R. A. Brown, "A constitutive equation for liquid crystalline polymer solutions", J. Rheol. 37 (1993), 413-441.

[3] C. V. Chaubal, L. G. Leal, and G. H. Fredrickson, "A Comparison of Closure Approximations for the Doi Theory of LCPs", J. Rheol. 39 (1995), 73-103.

[4] M. Doi, "Rheological properties of rodlike polymers in isotropic and liquid crystalline phases", Ferroelectrics 30 (1980), 247-254.

[5] M. Doi, "Molecular dynamics and rheological properties of concentrated solutions of rodlike polymers in isotropic and liquid crystalline phases", J. Polym. Sci. Polym. Phys. Ed. 19 (1981), 229-243.

[6] J. Feng and L. G. Leal, "Simulating complex flows of liquid-crystalline polymers using the Doi theory", J. Rheol. 41(6) (1997), 1317-1336.

[7] M. G. Forest and Q. Wang, "Biaxial patterns and director instabilities of lyotropic liquid crystalline polymers in imposed elongational flows", University of North Carolina Mathematics Department Preprint Series, \#97-14.

[8] M. G. Forest and Q. Wang, "Anisotropic microstructure-induced reduction of the Rayleigh instability for liquid crystalline polymers", Physics Letters A, to appear; "The role of microstructure in taming the Rayleigh instability of cylindrical jets", Physica D, accepted for special issue on Nonlinear Waves and Solitons in Physical Systems, editors R. Camassa and J. M. Hyman, to appear, 1998. 
[9] M. G. Forest, Q. Wang, and S. E. Bechtel, "1-D Models for thin filaments of liquid crystalline polymers: coupling of orientation and flow in the stability of simple solutions", Physica D 99 (1997), 527-554.

[10] T. Hu and G. Ryskin, "Numerical simulation of rodlike polymers in extensional and sink/source flows using the order-parameter version of Doi's theory", J. Chem. Phys. 96(6) (1992), 4705-4717.

[11] D. D. Joseph, Fluid dynamics of viscoelastic liquids, Springer-Verlag, New York, 1990.

[12] G. Marrucci, "Rheology of rodlike polymers in the nematic phase with tumbling or shear orientation", Macromolecules 24 (1991), 4176-4182.

[13] G. Marrucci and F. Greco, "Molecular structure of hedgehog point defects in nematics", Mol. Cryst. Liq. Cryst. 210 (1992), 129-141.

[14] Larson, R. G. and Öttinger, H, "Effect of molecular elasticity on out-of-plane orientations in shearing flows of liquid crystalline polymers", Macromolecules 24 (1991), 6270-6282.

[15] N. Mori, Y. Hamaguchi and K. Nakamura, "Numerical simulation of the spinning flow of liquid polymers", J. Rheol. 41 (5) (1997), 1095-1104.

[16] A. Rey, "Bifurcational analysis of the isotropic-nematic phase transition of rigid rod polymers subjected to biaxial stretching flow", Macromol. Theory Simul. 4(5) (1995), 857-872.

[17] H. See, M. Doi and R. G. Larson, "The effect of steady flow fields on the isotropic-nematic phase transition of rigid rod-like polymers", J. Chem. Physics 92(1) (1990), 792-800.

[18] Q. Wang, "Biaxial steady states and their stability in shear flows of liquid crystal polymers", J. Rheol. 41 (1997), 943-970. 


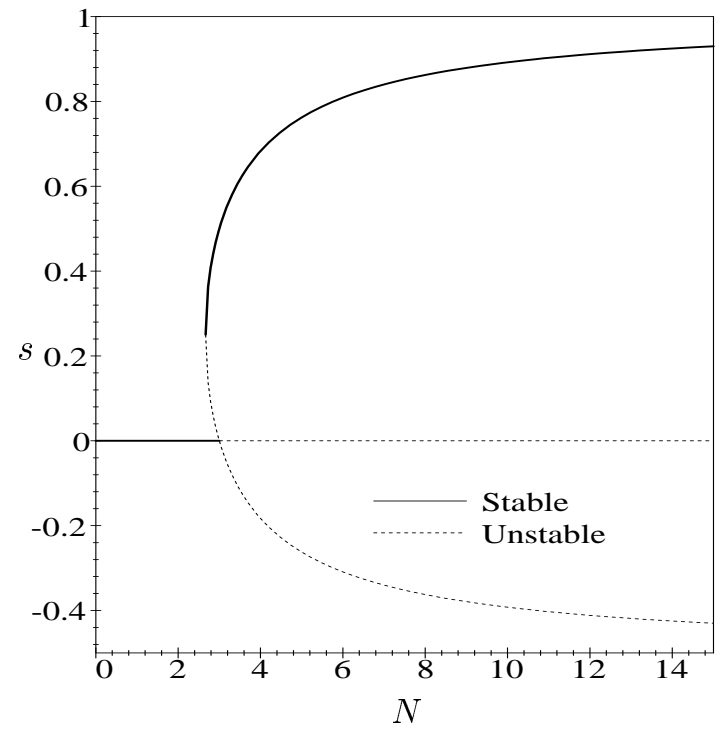

Figure 1: Uniaxial order parameter equilibria as functions of the polymer concentration $N$. The solid line represents the stable equilibria and the dotted line the unstable equilibria according to the Doi theory without an imposed flow field. 


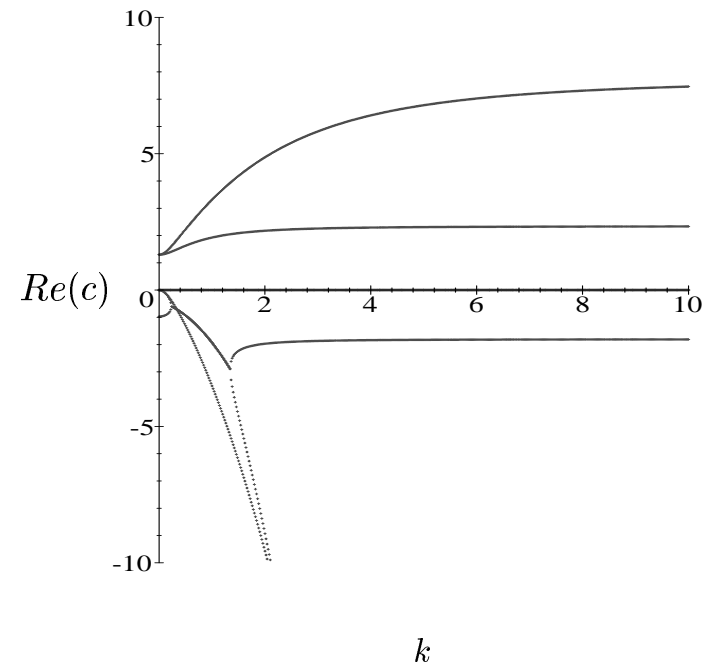

(a)

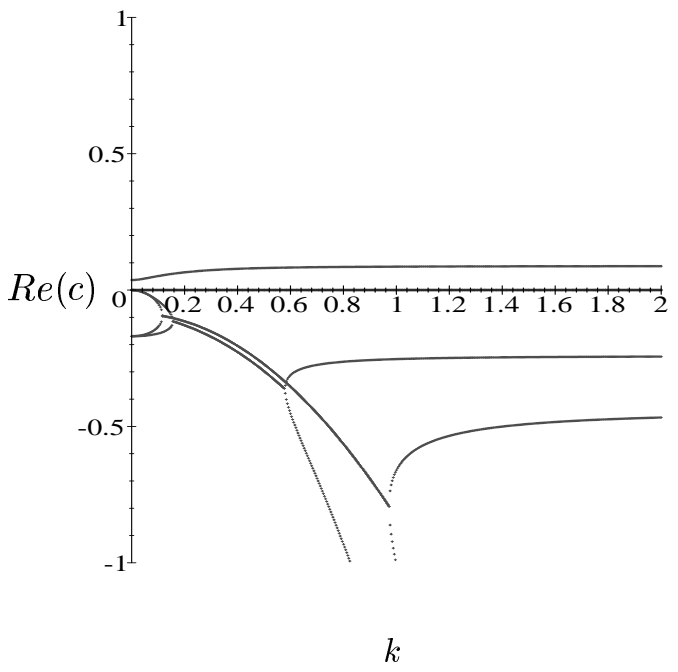

(b)

Figure 2: Growth, decay and neutral rates for unstable uniaxial equilibria in the spatially homogeneous LCP theory with solvent viscosity. Parameter values are $\sigma=0.7, \alpha=1, \lambda=$ $1, D r=0, \frac{1}{R e}=0.5$, and $\mathbf{k}=(k, k, k)$. (a). Oblate uniaxial equilibrium $s_{e q}<0$ for $N=6$. The two growth rates are bounded with a slight amplification over their zero wavevector limits. (b) Less-aligned prolate uniaxial equilibrium $0<s_{e q}<1 / 4$ for $N=2.8$. One growth rate persists for all wave numbers with a numerical value slightly above its zero wavevector limit. 


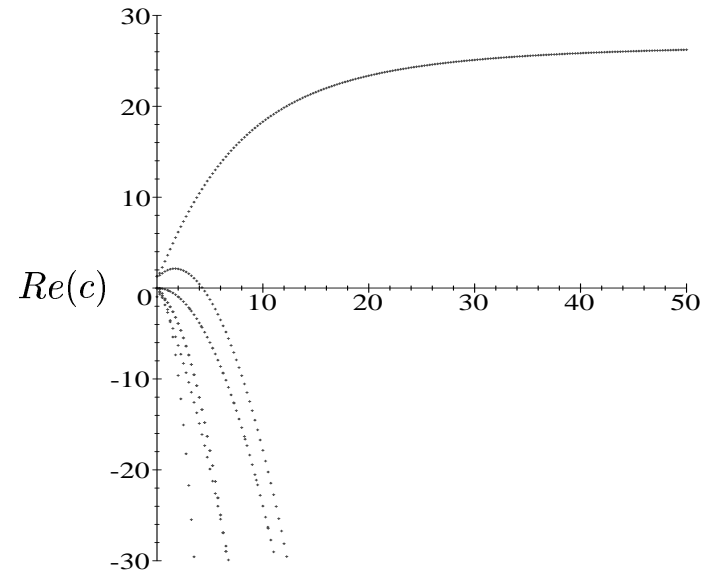

$k$

(a)

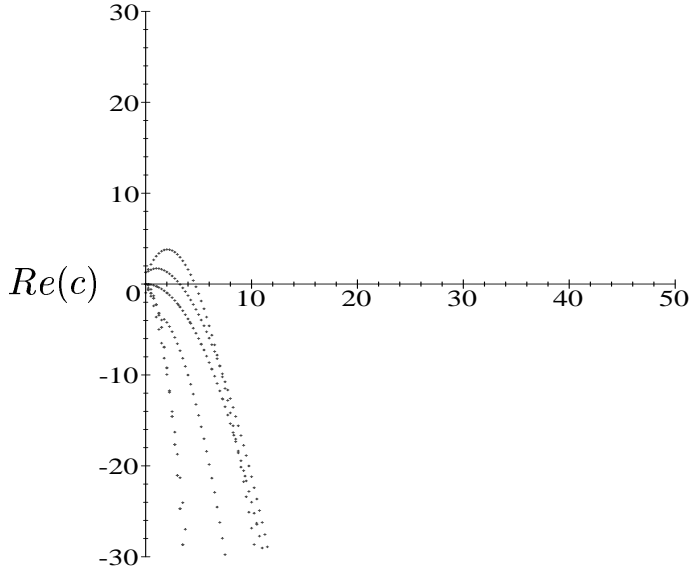

$k$

(b)

Figure 3: Growth, decay and neutral rates for the oblate uniaxial equilibrium with $N=6$ in the theory of LCPs with spatial inhomogeneity. Parameter values are $\sigma=0.7, \alpha_{h}=1, \lambda=$ $1, D r=0.1$, and $\mathbf{k}=(k, k, k)$. (a). Solvent viscosity is absent $\left(\frac{1}{R e}=0\right)$. One growth rate is restricted to long wavelengths, while the other growth rate persists for all $\mathbf{k}$, with a finite upper bound. (b) Solvent viscosity is included, $\frac{1}{R e}=0.5$, while all other parameters are identical to (a). Both growthrates have a finite wavelength cutoff. 


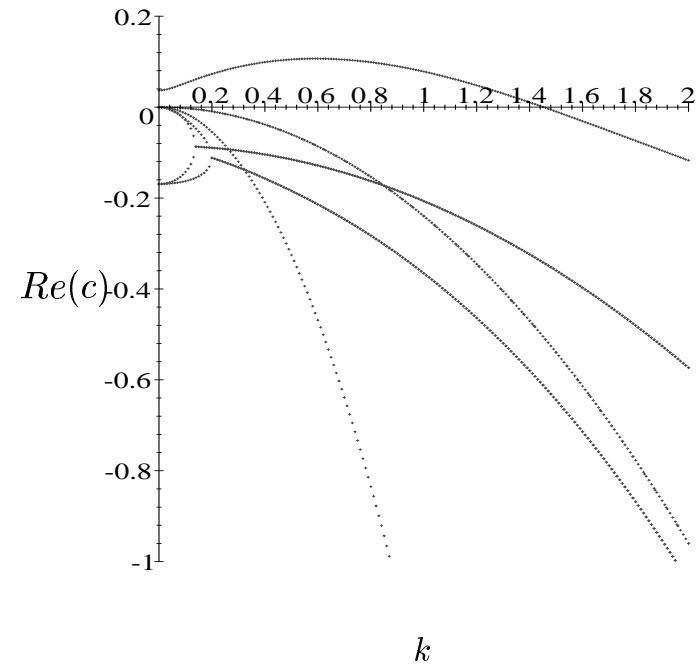

(a)

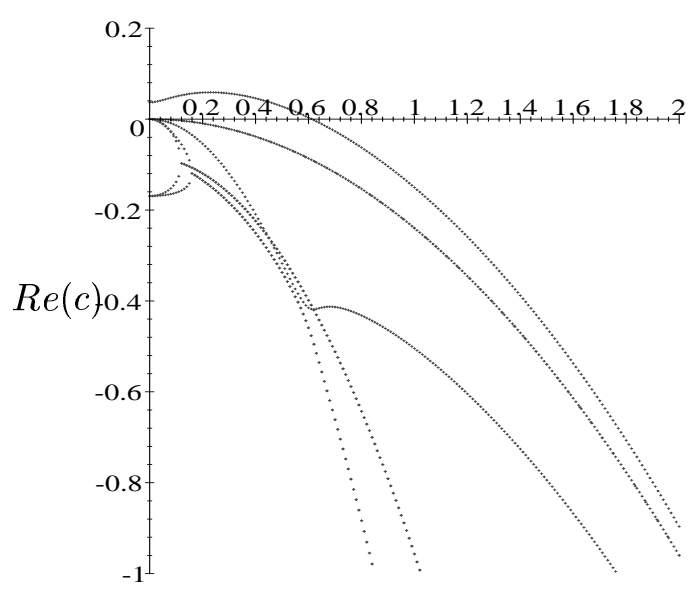

$k$

(b)

Figure 4: Growth, decay, and neutral rates for the less-aligned prolate equilibrium with $N=2.8$, for the theory of LCPs with spatial inhomogeneity. Parameter values are $\sigma=$ $0.7, \alpha_{h}=1, \lambda=1, D r=0.1$, and $\mathbf{k}=(k, k, k)$. (a). Solvent viscosity is absent, $\left(\frac{1}{R e}=0\right)$. The only growth rate has a finite wavelength cutoff. (b) Solvent viscosity is included, $\frac{1}{R e}=0.5$, with all other parameters as in (a). There is no qualitative change, rather all rates are quantitatively reduced. 